\title{
Acknowledgment of Reviewers, 2018
}

The success of Phytopathology depends on the quality of manuscripts submitted by authors and on the care and competence with which they are reviewed. It is the policy of the Editorial Board to solicit reviews for manuscripts from specialists who are most qualified to review them. In addition to members of the Editorial Board, the individuals listed below provide constructive critical reviews of one or more manuscripts during the past year. Their names are published here in grateful appreciation of their contribution to the journal and to the science of phytopathology. http://dx.doi.org/10.1094/PHYTO-109-1-0004

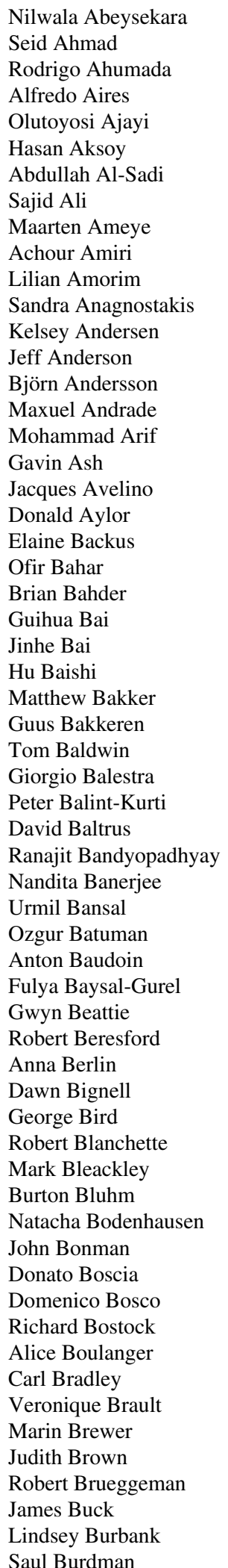

Nilwala Abeysekara

Seid Ahmad

Alfredo Aires

Olutoyosi Ajayi

Hasan Aksoy

Abdullah Al-Sadi

Lilian Amorim

Sandra Anagnostakis

Kelsey Andersen

Maxuel Andrade

Mohammad Arif

in Ash

Elaine Backus

Ofir Bahar

Jinhe Bai

Hu Baish

Matthew Bakker

Guus Bakkeren

Peter Balint-Kurti

David Baltrus

Ranajit Bandyopadhyay

ita Banerjee

Anton Baudoin

Fulya Baysal-Gurel

Anna Berlin

Dawn Bignell

George Bird

Robert Blanchette

Natacha Bodenhausen

John Bonman

Donato Boscia

Alice Boulanger

Carl Bradley

Veronique Brault

Robert Brueggeman

Lindsey Burbank

Saul Burdman
Treena Burgess

Lenka Burketova

Elliott Bussell

Lance Cadle-Davidson

Atilio Castagnaro

Antonio Castillo

Pablo Castillo

Sophie Cesbron

Chung Chang

Daniel Chapman

Nikki Charlton

Subhadeep Chatterjee

Jianchi Chen

Jianli Chen

Weidong Chen

Wen Chen

Xianming Chen

Xiaoren Chen

Kuo-Szu Chiang

Martin Chilvers

Robin Choudhury

Chia-Lin Chung

Maisa Ciampi-Guillardi

Christopher A. Clark

Jeffery Coleman

Bret Cooper

Ted Cottrell

Christina Cowger

Andrew Craig

Jaime Cubero

Mirna Curkovic-Perica

Giusy D'Attoma

James Dale

Felipe Dalla Lana

Louise-Marie Dandurand

Ciara Dangerfield

Leo de la Fuente

Alessandra de Souza

Paul De Vos

Giuliano Degrassi

Emerson Del Ponte

Xiaoling Deng

Veena Devi Ganeshan

Megan Dewdney

Francesco Di Serio

Juan Diaz Ricci

Juan Antonio Díaz-Pendón

Donald Dickson

Leslie Domier

Ruairi Donnelly

Anne Dorrance

Greg Douhan

Vinson Doyle

Peter Dracatos

Ibrahim Draz

Tanja Dreo

Martin Drucker

Shuo Duan

Y. Duan

Bojan Duduk
Nicholas Dufault

Korsi Dumenyo

Jeremiah Dung

Cyril Dutech

Bhabesh Dutta

Daniel Ebbole

Noam Eckshtain-Levi

Martin Egan

Jon Eisenback

Paul Esker

Kathy Esvelt Klos

Katherine Evans

Sydney Everhart

Frédéric Fabre

Mark Farman

Chunda Feng

Christopher Ference

Marc Fermaud

Dilantha Fernando

Francis Ferrandino

Marta Filippi

Greg Forbes

Bruna Forcelini

Paul Fourie

Bart Fraaije

Isolde Francis

Rudolph Fredua-Agyeman

Joshua Freeman

Timothy Friesen

Kenneth Frost

William Fry

David Gadoury

László Galgóczy

Jean Luc Gallois

San-Ji Gao

Matteo Garbelotto

Paolina Garbeva

Andrew Geering

Murad Ghanim

Annalisa Giampetruzzi

Michel Giraud

Pierre Gladieux

Anthony Glenn

Cláudia Godoy

Hossein Golzar

Tom Gordon

Erica Goss

Bruce Gossen

Sven Gottwald

Francine Govers

Aska Goverse

Zane Grabau

Thomas Gradziel

Jim H. Graham

Sarah Green

Michael Grisham

Rita Grosch

Rebecca Grumet

Niklaus Grünwald

Maria Lodovica Gullino
Shiwei Guo

Abolfazl Hajihassani

Reza Hajimorad

Susan Halbert

Adam Hall

Jong Hyun Ham

Frédéric Hamelin

John Hammond

Rosemarie Hammond

Everett Hansen

Inge Hanssen

Guixia Hao

Scott Harper

John Hartung

Katsunori Hatakeyama

Dwayne Hegedus

Tarek Hewezi

Mark Hilf

Angelyn Hilton

Kevin Hockett

Dave Hooker

Jeff Hoy

Xiaoping $\mathrm{Hu}$

Julio Huerta-Espino

Sonia Humphris

Mario Inomoto

Toru Iwanami

Anjali Iyer-Pascuzzi

Marie-Agnes Jacques

Wojciech Janisiewicz

Chatchawan Jantasuriyarat

Andrew Jarosz

Guanghai Ji

Yulin Jia

Daohong Jiang

Dennis Johnson

Evan Johnson

Jeffrey Jones

John Jones

Richard Jones

Ramon Jordan

Annemarie Justesen

Prem Kandel

Yuba Kandel

Zhensheng Kang

Alexander Karasev

Matthew Kasson

Anthony Keinath

Jim Kerns

Churamani Khanal

Chang Hyun Khang

Sebastian Kiewnick

Nabil Killiny

Dae-Hyuk Kim

Yong-Ki (Richard) Kim

Abby Kinchy

Anna Klimes

Steven Klosterman

Dan Kluepfel

Jessica Koczan 
Linda Kohn

Frederic Kolb

Judit Komáromi

Alissa Kriss

Rodrigo Krugner

Gretchen Kuldau

Sridhara Kunjeti

Nemanja Kuzmanović

Blanca Landa

David Langston

francisco Larajeira

Robert Larkin

Kathy Lawrence

Bruno Le Cam

Jared Leboldus

Je Min Lee

John Leslie

Amit Levy

Julien levy

Melanie Lewis Ivey

Jinyun $\mathrm{Li}$

Hong Lin

Steven Lindow

Kai-Shu Ling

Christopher Little

Ke Liu

Michele Loewen

Marianne Loiseau

Joao Lopes

Juan Jose Lopez-Moya

Tiffany Lowe-Power

Chao-Xi Luo

Jing Luo

Zhonghua Ma

Laurence Madden

Walt Mahaffee

Michael Malnoy

Kranthi Mandadi

Gelsomina Manganiello

Liangang Mao

Robert Marra

Potrykus Marta

Frank Martin

Shin-Yi Marzano

Alex Mastin

Anna Maria Mastrangelo

Dmitri Mavrodi

Margaret McGrath

Robert McIntosh

Neil McRoberts

Alistair McTaggart

Angela Mehta

Rebecca Melanson

Ulrich Melcher

Artemio Mendoza-Mendoza

Melody Meyer-Jertberg

Marcel Meyer

Themis J. Michailides

Phillip Miklas

Sally Miller

Alice Milne

Charles Mitchell

Eduardo Mizubuti

Massimiliano Morelli

Mathew Morra

Mathieu Moslonka-Lefebvre

Daren Mueller

Anja Muller

Chris Mundt
Gary Munkvold

Alejandra Munoz Bodnar

Rita Musetti

Beatriz Navarro

Jesús Navas-Castillo

Jonathan Negrel

Dianne Newman

Henry Ngugi

Zachary Noel

Maria Teresa Novo-Mansur

Leonard Nunney

Kerry O'Donnell

James Oard

Ken Obasa

Denis Odokonyero

Francis Ogbonnaya

Kouhei Ohnishi

Ricardo Oliva

Jonathan Oliver

Richard Oliver

Elena Orellano

Viviana Ortiz

Manuel Ospina-Giraldo

Israel Pagán

Franck Panabières

Bernd Panassiti

Sheo Shankar Pandey

Zhiqian Pang

Epaminondas Paplomatas

Mathews Paret

Nicolas Parisey

Pierce Paul

Timothy Paulitz

You-Liang Peng

Natalia Peres

Sambasivam Periyannan

Elena Perry

Gary Peterson

María Julia Pianzzola

Hans-Pieter Piepho

Elizabeth Pierson

Minna Pirhonen

Andrew Pitman

Randy Ploetz

Jesse Poland

James Polashock

Anissa Poleatewich

Sudarsana Poojari

Lyndon Porter

Thomas Powers

Simone Prospero

Dov Prusky

Alexander Purcell

Alexander Putman

Pan Qinghua

Wenping Qiu

Feng Qu

Mahbubjon Rahmatov

Oscar Ramos

Jennifer Randall

Arash Rashed

Nidhi Rawat

Jean-Sébastien Reynard

Camila Ribeiro

Nicolas Rispail

Mark Ritenour

Cécile Robin

Jeffrey Rollins

Gianfranco Romanazzi
Caroline Roper

Vittorio Rossi

Matthew Rouse

Megan Rúa

Will Rutter

Seiya Saito

Pasquale Saldarelli

Ahmad Sallam

Berit Samils

Erzsébet Sándor

Hyunkyu Sang

Maria Saponari

Diane Saunders

Arthur Schaafsma

Leonardo Schena

Harald Scherm

Rebecca Schewe

Herman Scholthof

Henk-Jan Schoonbeek

Brenda Schroeder

Nathan Schroeder

Marco Scortichini

Jason Scott

Mamoudou Setamou

Paul Severns

Weixing Shan

Gregory Shaner

Roshan Sharma Poudel

Brian Shaw

Michael Shaw

Nina Shishkoff

Dani Shtienberg

Anne Sicard

Raksha Singh

Ravi Singh

Mark Sisterson

Stephanie Slinski

Jadwiga Śliwka

Theo Smits

Peter Solomon

Adam Sparks

Christopher Steel

Ioannis Stergiopoulos

Ethan Stewart

Jane Stewart

Lucy Stewart

Jeffrey Stone

Krishna Subbarao

Rajgopal Subramaniam

Mysore Sudarshana

Haruhisa Suga

Sunitha Sukumaran

Karen Sullam

George Sundin

Nobuhiro Suzuki

Bryan Swingle

Anna Szyniszewska

Firas Talas

Yanglan Tan

Adam Taranto

Paul Taylor

Francisco Tenllado

Marco Thines

Carla Thomas

Jennifer Thompson

Paul Tooley

Lesley Torrance

Yukio Tosa

Gregory Tylka
Ioannis Tzanetakis

William Underwood

Lisa Vaillancourt

Miroslav Valárik

Gary Vallad

C. Eduardo Vallejos

Marc Valls

Theo van der Lee

Jacquie van der Waals

Wopke van der Werf

Jan van der Wolf

Frederique Van Gijsem

Jan van Kan

Joel Vanneste

Thomas Vatter

Antonio Vicent

Ivana Vico

Sara Villani

Boris Vinatzer

Botma Visser

Andreas von Tiedemann

Anne-Sophie Walker

Jie Wang

Meinan Wang

Nian Wang

Yiming Wang

Astri Wayadande

Melanie Weckert

Vance Whitaker

Steven White

Jean Williams-Woodward

Laetitia Willocquet

Richard Wilson

Ted Wilson

William Wintermantel

James Woodhall

Bo Ming Wu

Nelson Wulff

Stephen Wylie

Katia Xavier

Jiatao Xie

Jin-Rong Xu

Mingliang Xu

Xiangming $\mathrm{Xu}$

Qing Yan

Ching-Hong Yang

Li Yang

Wenwu Ye

Raymond Yokomi

Anthony Young

Meng Yuan

Alex Zaccaron

Paulo Zaini

Inga Zasada

Quan Zeng

Jiasui Zhan

Aimin Zhang

Lei Zhang

Shujian Zhang

Xiaoyu Zhang

Yucheng Zhang

Yunzeng Zhang

Bingyu Zhao

Shaobin Zhong

Bo Zhou

Laura Ziems

Carmit Ziv 